Communications in Physics, Vol. 23, No. 3 (2013), pp. 235-240

\title{
INFLUENCE OF SPIN FLUCTUATION ON THE MAGNETIC PROPERTIES OF EUO ULTRA-THIN FILM
}

\author{
PHAM HUONG THAO \\ Faculty of Physics, Hue College of Education, \\ 32 Le Loi, Thua Thien Hue, Vietnam \\ BACH THANH CONG \\ Faculty of Physics, VNU University of Science, \\ 334 Nguyen Trai, Thanh Xuan Hanoi, Vietnam \\ Email: congbachthanh@gmail.com \\ Received 25 June 2013 \\ Accepted for publication 15 September 2013
}

\begin{abstract}
The Gaussian spin fluctuation theory is applied to study magnetic properties (Curie temperature, magnetization) of EuO ultra-thin films within nearest neighbor and next nearest neighbor exchange approximation. The dependence of Curie temperature on the thickness of the free EuO ultrathin film is calculated and compared with the results of the other mean field methodconstant coupling approximation. The effect of nearly independence of saturation magnetization on the EuO films thickness measured in [8] can be explained by controlling the next nearest neighbor exchange in presence of substrates.
\end{abstract}

\section{INTRODUCTION}

In the recent years, magnetic properties of thin films which have been intensively investigated by both theoretical and experimental methods expose a great potential for applications [1]. The important reasons of magnetic size effects in the thin films, such as the reduction of the Curie temperature $T_{C}$, are the finite thickness and the broken translational symmetry along the direction perpendicular to the film surfaces. Many theoretical methods are used to study the magnetic properties of thin films such as, the standard mean field (MF) methods [2] including the Weiss mean field, the Oguchi's cluster and the constant coupling approximations (CCA), spin wave method [3], and recently, the integral functional integral method [4]- [5]. Our paper is aimed to apply the Gaussian spin fluctuation (SF) theory developed by us [4] for a real spin system of $\mathrm{EuO}$ thin film. Here we consider the next nearest neighbor (NNN) exchange parallel with the nearest neighbor $(\mathrm{NN})$ exchange interactions between spins for explanation of some special effects in EuO. $\mathrm{EuO}$ has low magnetic anisotropy, the spin of the $\mathrm{Eu}^{2+}$ is localized with value $S=7 / 2$. Ultrathin EuO film is used as tunnel barriers in semiconductor spintronic devices and its large exchange splitting is investigated in [6]. Europium monoxide is nearly an ideal Heisenberg ferromagnet [6], so it is a good case for testing theories for thin films. In the 
second part we give some essential details of the model and the functional integral method (FIM) applied for ultrathin films.

\section{BRIEF THEORY}

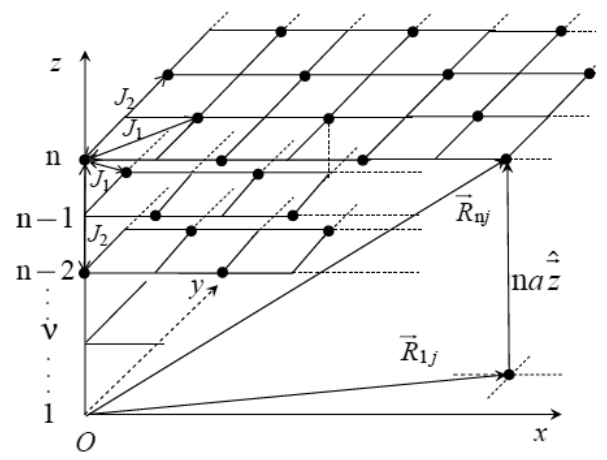

Fig. 1. A ferromagnetic thin film of the face centered cubic lattice. The spin position is defined by index $\nu j$, where $\nu$ is a plane index $\nu=1,2, \ldots, n$ and $j$ stands for a two-dimensional lattice vector $\vec{R}_{j}$ in $x O y$ plane parallel to the film surfaces. $J_{1}\left(J_{2}\right)$ is the $\mathrm{NN}(\mathrm{NNN})$ exchange integral.

We consider a thin film which has a face centered cubic (fcc) spin lattice comprising from n layers and there are $N$ spins in every layer (Fig. 1). The Heisenberg Hamiltonian for the spin films, when the mean field is included in non-interacting Hamiltonian, is written as:

$$
\begin{aligned}
H & =H_{0}+H_{i n t} \\
H & =-g \mu \sum_{\nu j} h_{\nu} S_{\nu j}^{z}-\frac{1}{2} \sum_{\nu j, \nu^{\prime} j^{\prime}} J_{\nu \nu^{\prime}}\left(\vec{R}_{j}-\vec{R}_{j^{\prime}}\right) \delta \vec{S}_{\nu j} \delta \vec{S}_{\nu^{\prime} j^{\prime}}
\end{aligned}
$$

with

$$
\begin{gathered}
h_{\nu}=h+(g \mu)^{-1} \sum_{\nu^{\prime}} J_{\nu \nu^{\prime}}(0)\left\langle S_{\nu^{\prime}}^{z}\right\rangle \\
\delta S_{\nu j}^{z}=S_{\nu j}^{z}-\left\langle S_{\nu^{\prime}}^{z}\right\rangle ; \delta S_{\nu j}^{x}=S_{\nu j}^{x} ; \delta S_{\nu j}^{y}=S_{\nu j}^{y} ; \alpha=x, y, z \\
J_{\nu \nu^{\prime}}(\vec{k})=\sum_{j-j^{\prime}} J_{\nu \nu^{\prime}}\left(\vec{R}_{j}-\vec{R}_{j^{\prime}}\right) \exp \left[i \vec{k}\left(\vec{R}_{j}-\vec{R}_{j^{\prime}}\right)\right]
\end{gathered}
$$

where the first term of (1) is the spin energy in the layer-dependent field $h_{\nu}$ consisting of the external field $h$ and molecular (Weiss) field. The second term of (1) is the usual Heisenberg exchange interaction between spin fluctuations $\delta \vec{S}_{\nu j}$ and $\delta \vec{S}_{\nu^{\prime} j^{\prime}}$, which have three components described by $(3) . J_{\nu \nu^{\prime}}\left(\vec{R}_{j}-\vec{R}_{j^{\prime}}\right)$ denotes the exchange integral between spin fluctuations, which is considered as the matrix element of the matrix $\hat{J}\left(\vec{R}_{j}-\vec{R}_{j^{\prime}}\right)$. $J_{\nu \nu^{\prime}}(0)$ is Fourier image of exchange integral taken at $\vec{k}=0$ (see (4)).

According to [4], the free energy of the magnetic film in the Gaussian approximation has the following form: 


$$
\begin{aligned}
F & =\frac{N}{2} \sum_{\nu, \nu^{\prime}} J_{\nu \nu^{\prime}}(0)\left\langle S_{\nu}^{z}\right\rangle\left\langle S_{\nu^{\prime}}^{z}\right\rangle-\frac{N}{\beta} \sum_{\nu} \ln \frac{\operatorname{sh}(S+1 / 2) y_{\nu}}{\operatorname{sh} \frac{y_{\nu}}{2}} \\
& +\frac{1}{2 \beta} \sum_{\vec{k}} \ln \operatorname{det}|\hat{1}-\hat{A}(\vec{k})|+\frac{1}{\beta} \sum_{\vec{k}, \omega} \ln \operatorname{det}|\hat{1}-\hat{B}(\vec{k}, \omega)|
\end{aligned}
$$

Here $\hat{A}(\vec{k})$ and $\hat{B}(\vec{k}, \omega)$ are $\mathrm{n} \times \mathrm{n}$ matrices, which have the following form when the NN and NNN exchange interactions between spin fluctuation are taken into account:

$$
\hat{A}(\vec{k})=\left(\begin{array}{cccccc}
\beta b^{\prime}\left(y_{1}\right) J_{s}(\vec{k}) & \beta b^{\prime}\left(y_{1}\right) J(\vec{k}) & \beta b^{\prime}\left(y_{1}\right) J_{0}(\vec{k}) & 0 & \ldots & 0 \\
\beta b^{\prime}\left(y_{2}\right) J(\vec{k}) & \beta b^{\prime}\left(y_{2}\right) J_{s}(\vec{k}) & \beta b^{\prime}\left(y_{2}\right) J(\vec{k}) & \beta b^{\prime}\left(y_{2}\right) J_{0}(\vec{k}) & \ldots & 0 \\
\beta b^{\prime}\left(y_{3}\right) J_{0}(\vec{k}) & \beta b^{\prime}\left(y_{3}\right) J(\vec{k}) & \beta b^{\prime}\left(y_{3}\right) J_{s}(\vec{k}) & \beta b^{\prime}\left(y_{3}\right) J(\vec{k}) & \ldots & 0 \\
0 & \beta b^{\prime}\left(y_{4}\right) J_{0}(\vec{k}) & \beta b^{\prime}\left(y_{4}\right) J(\vec{k}) & \beta b^{\prime}\left(y_{4}\right) J_{s}(\vec{k}) & \ldots & 0 \\
\vdots & \vdots & \vdots & \vdots & \vdots & \vdots \\
0 & 0 & 0 & 0 & \ldots & \beta b^{\prime}\left(y_{n}\right) J_{s}(\vec{k})
\end{array}\right)
$$

and

$$
\hat{B}(\vec{k}, \omega)=\left(\begin{array}{cccccc}
\frac{\beta b\left(y_{1}\right) J_{s}(\vec{k})}{y_{1}-i \beta \omega} & \frac{\beta b\left(y_{1}\right) J(\vec{k})}{y_{1}-i \beta \omega} & \frac{\beta b\left(y_{1}\right) J_{0}(\vec{k})}{y_{1}-i \beta \omega} & 0 & \ldots & 0 \\
\frac{\beta b\left(y_{2}\right) J(\vec{k})}{y_{2}-i \beta \omega} & \frac{\beta b\left(y_{2}\right) J_{s}(\vec{k})}{y_{2}-i \beta \omega} & \frac{\beta b\left(y_{2}\right) J(\vec{k})}{y_{2}-i \beta \omega} & \frac{\beta b\left(y_{2}\right) J_{0}(\vec{k})}{y_{2}-i \beta \omega} & \ldots & 0 \\
\frac{\beta b\left(y_{3}\right) J_{0}(\vec{k})}{y_{3}-i \beta \omega} & \frac{\beta b\left(y_{3}\right) J(\vec{k})}{y_{3}-i \beta \omega} & \frac{\beta b\left(y_{3}\right) J_{s}(\vec{k})}{y_{3}-i \beta \omega} & \frac{\beta b\left(y_{3}\right) J(\vec{k})}{y_{3}-i \beta \omega} & \ldots & 0 \\
0 & \frac{\beta b\left(y_{4}\right) J_{0}(\vec{k})}{y_{4}-i \beta \omega} & \frac{\beta b\left(y_{4}\right) J(\vec{k})}{y_{4}-i \beta \omega} & \frac{\beta b\left(y_{4}\right) J_{s}(\vec{k})}{y_{4}-i \beta \omega} & \ldots & 0 \\
\vdots & \vdots & \vdots & \vdots & \vdots & \vdots \\
0 & 0 & 0 & 0 & \ldots & \frac{\beta b\left(y_{n}\right) J_{s}(\vec{k})}{y_{n}-i \beta \omega}
\end{array}\right)
$$

with

$$
\begin{gathered}
y_{\nu}=\beta g \mu h_{\nu} \\
\omega=2 \pi l / \beta ; l=0, \pm 1, \pm 2, \ldots
\end{gathered}
$$

Longitudinal and transversal spin fluctuation amplitudes can be calculated through the correlation functions of the spin fluctuation components (or time-ordered spin Green functions) as follows:

$$
\begin{aligned}
& \chi_{\nu_{1} j_{1}, \nu_{2} j_{2}}\left(\tau_{1}, \tau_{2}\right)=\chi_{\nu_{1} \nu_{2}}^{z}\left(\vec{R}_{j_{1}}-\vec{R}_{j_{2}}, \tau_{1}-\tau_{2}\right) \\
&=\left\langle\hat{T}\left(S_{\nu_{1} j_{1}}^{z}\left(\tau_{1}\right)-\left\langle S_{\nu_{1}}^{z}\right\rangle\right)\left(S_{\nu_{2} j_{2}}^{z}\left(\tau_{2}\right)-\left\langle S_{\nu_{2}}^{z}\right\rangle\right)\right\rangle \\
& \chi_{\nu_{1} j_{1}, \nu_{2} j_{2}}^{x}\left(\tau_{1}, \tau_{2}\right)=\chi_{\nu_{1} \nu_{2}}^{x}\left(\vec{R}_{j_{1}}-\vec{R}_{j_{2}}, \tau_{1}-\tau_{2}\right)=\left\langle\hat{T} S_{\nu_{1} j_{1}}^{x}\left(\tau_{1}\right) S_{\nu_{2} j_{2}}^{x}\left(\tau_{2}\right)\right\rangle \\
& \chi_{\nu_{1} j_{1}, \nu_{2} j_{2}}^{y}\left(\tau_{1}, \tau_{2}\right)=\chi_{\nu_{1} \nu_{2}}^{y}\left(\vec{R}_{j_{1}}-\vec{R}_{j_{2}}, \tau_{1}-\tau_{2}\right)=\left\langle\hat{T} S_{\nu_{1} j_{1}}^{y}\left(\tau_{1}\right) S_{\nu_{2} j_{2}}^{y}\left(\tau_{2}\right)\right\rangle
\end{aligned}
$$


Within the Gaussian approximation the Fourier image of the spin correlation function (10) becomes :

$$
\tilde{\chi}(\vec{k}, \omega)=-\frac{1}{\beta}\left[J_{\nu_{1} \nu_{2}}^{-1}(\vec{k})-\sum_{\nu} J_{\nu_{1} \nu}^{-1}(\vec{k}) C_{\nu_{1} \nu_{2}}^{\alpha}(\vec{k}, \omega)\right]
$$

where $C_{\nu \nu^{\prime}}^{\alpha}(\vec{k}, \omega)$ is the elements of the matrix $\hat{C}(\vec{k}, \omega)$ and

$$
\hat{C}^{z}(\vec{k}, \omega)=\hat{C}^{z}(\vec{k})=(\hat{1}-\hat{A}(\vec{k}))^{-1} ; \hat{C}^{x(y)}(\vec{k}, \omega)=(\hat{1}-\hat{B}(\vec{k}, \omega))^{-1} ;
$$

The relative magnetic moment per site of the $\nu^{t h}$ spin layer in the mean field approximation (MFA) and spin fluctuation approximation (SFA) has the following forms:

$$
\begin{gathered}
m_{\nu}^{M F}(\beta)=\left\langle S_{\nu}^{z}\right\rangle_{0}=b\left(y_{\nu}\right) \\
m_{\nu}^{S F}(\beta)=\left\langle S_{\nu}^{z}\right\rangle=\left\{S(S+1)-\frac{1}{N} \sum_{\alpha, \vec{k}, \omega}\left(\chi_{\nu \nu}^{\alpha \alpha}(\vec{k}, \omega)\right)\right\}^{1 / 2}
\end{gathered}
$$

In order to compare with experiments and other methods, an average relative (or dimensionless) magnetic moment per thin film site is given as

$$
m(\beta)=\frac{M}{\mu N n}=\frac{1}{n} \sum_{\nu=1} n m_{\nu}(\beta)
$$

Average magnetizations in the MF and SF approximations are denoted by $m^{M F}$ and $m^{S F}$, respectively.

\section{NUMERICAL RESULTS AND DISCUSSION}

The Fourier components of exchange integrals are easily derived from the geometrical structure of fcc thin film spin lattice as:

$$
J_{s}(\vec{k})=J_{s_{1}}(\vec{k})+J_{s_{2}}(\vec{k})
$$

with $J_{s_{1}}(\vec{k})=4 J_{1} \cos \left(\frac{k_{x} a}{2}\right) \cos \left(\frac{k_{y} a}{2}\right)$ and $J_{s_{1}}(\vec{k})=2 J_{2}\left[\cos \left(k_{x} a\right)+\cos \left(k_{y} a\right)\right]$.

$$
\begin{gathered}
J(\vec{k})=2 J_{2}\left[\cos \left(\frac{k_{x} a}{2}\right)+\cos \left(\frac{k_{y} a}{2}\right)\right] \\
J_{0}(\vec{k})=J_{2}
\end{gathered}
$$

where $a$ is a lattice constant of the thin film. In the following parts, a relative dimensionless temperature $\tau=\frac{T}{T_{C}^{b}}$ is used. $T_{C}^{b}$ is the Curie temperature of the corresponding bulk spin lattice, in which $T_{C}^{b}=\frac{S(S+1) Z J}{3 k_{B}}$ ( $Z$ is the number of NN spins, for fcc spin lattice, $Z=12$ ). The Curie temperature within the MF (SF) theory is obtained from a solution of an equation $m^{M F}\left(\tau_{C}\right)=0\left(m^{S F}\left(\tau_{C}\right)=0\right)$, and expressed by $\tau_{C}=\frac{T_{C}}{T_{C}^{b}}$.

The numerical calculations for the fcc $\mathrm{EuO}$ free thin film spin lattice at zero external magnetic field $(h=0)$ have been performed with following parameter values $[2]: S=7 / 2$, 
$J_{1} / k_{B}=0.606 K, J_{2} / k_{B}=0.119 K$. Table 1 presents the comparison of our results for $T_{C}$ of EuO free thin film within the MFA and the SFA with ones of R. Rausch and W. Nolting in the CCA [2]. Since EuO has a low anisotropy, it can obey the Mermin-Wagner theorem [7]. Our SFA values for are lower than ones of MFA and CCA and agree better with the Mermin-Wagner theorem. Moreover, CCA gave unclear values of $T_{C}$ when the thickness of the thin film larger 5 atomic layers (these values larger the Curie temperature for bulk $\left.\mathrm{EuO}, T_{C}^{b} \approx 69 K\right)$.

Table 1. Comparison of values of the Curie temperature $T_{C}(\mathrm{n})$ which are calculated for EuO within MFA, SFA with ones within the CCA [2].

\begin{tabular}{cccc}
\hline \multicolumn{4}{c}{$T_{C}(\mathrm{n})(\mathrm{K})$} \\
\hline $\mathrm{n}$ & $\mathrm{MFA}$ & $\mathrm{SF}$ & $\mathrm{CCA}$ \\
\hline 1 & 17.12 & 3.40 & 22.70 \\
2 & 31.54 & 10.12 & 50.00 \\
3 & 36.15 & 13.57 & 61.10 \\
4 & 40.27 & 16.33 & 66.60 \\
5 & 43.89 & 19.13 & 69.60 \\
8 & 52.25 & - & 73.60 \\
\hline
\end{tabular}

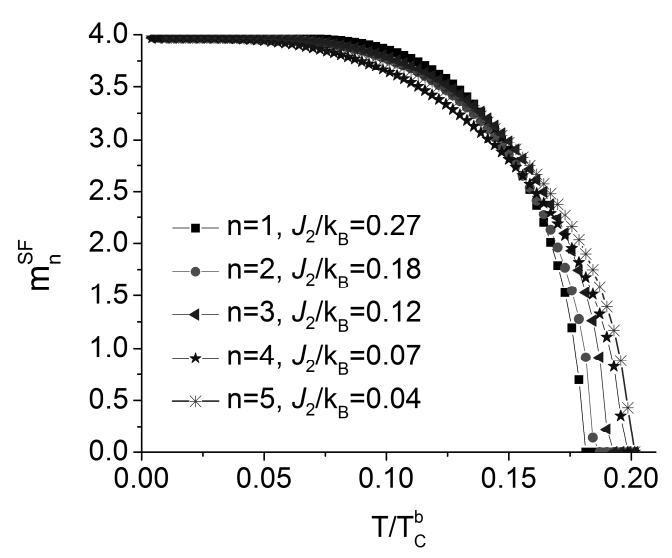

Fig. 2. The magnetization of EuO ultra-thin films with different thicknesses and NNN exchange integral as a function of temperature in SFA.

Our results given in the SF theory for free $\mathrm{EuO}$ films are much smaller than the experimental ones for $\mathrm{EuO}$ films on substrates [8]. The reason can be the influence of the substrates on the exchange interaction between the spins of $\mathrm{EuO}$ thin films. The substrates can make the Curie temperature of the thin films increase (or decrease). According to Ezana Negusse et al. [8], when EuO films were grown as a stepped wedge on $\mathrm{Si} / \mathrm{SiO} 2 / \mathrm{Cr}(20 \AA) / \mathrm{Cu}(90 \AA)$ and capped with $\mathrm{Y}(20 \AA) / \mathrm{Al}(80 \AA)$, the film high quality and consistent magnetic properties were confirmed by superconducting quantum interference device magnetometry, which revealed a constant saturation moment independent of film 
thickness. This phenomenon may be explained by increasing the role of NNN exchange $J_{2}$, which is in longer range than $J_{1}$, with reduction of the film thicknesses. Therefore, we fix the NN exchange integral and modify the NNN exchange integral $J_{2}$ (see Fig. 2).

From Fig. 2, one can see that the Curie temperature change only about $12 \%$ and maximum change of saturation magnetization below Curie temperature is about $3 \%$ when the film thicknesses reduce from 5 atomic layer to monolayer. This result gives the reasonable explanation for the experimental observation [8] that the saturation magnetization of $\mathrm{EuO}$ thin film is nearly independent on the reduction of the film's thickness.

\section{CONCLUSION}

The FIM for solving Heisenberg model in quasi two dimensional case is applied to find the magnetization, Curie temperature as a function of temperature, thickness and exchange interaction of the $\mathrm{EuO}$ ultrathin films with and without the substrate. Our results for free $\mathrm{EuO}$ films given in SFA are more appropriate to Mermin-Wagner theorem than ones of CCA. In addition, we have shown that the effect of the nearly independence of the saturation magnetization on the films thickness observed experimentally for $\mathrm{EuO}$ thin films may be explained by enhancing the influence of NNN exchange interaction.

\section{ACKNOWLEDGMENTS}

This research is funded by Vietnam National Foundation for Science and Technology Development (NAFOSTED) under grant number 103.02.2012.37.

\section{REFERENCES}

[1] John P. Volkerts, Magnetic Thin Films: Performance and Applications, Nova Science Publishers, Incorporated, 2011.

[2] Roman Rausch and Wolfgang Nolting, J. Phys.: Condens. Matter 21 (2009) 376002.

[3] V. Yu. Irkhin, A. A. Katanin, and M. I. Katsnelson, Phys. Rev. B 60 (1999) 1082.

[4] Bach Thanh Cong, Pham Huong Thao, Physica B 426 (2013) 144149.

[5] Bach Thanh Cong, Pham Huong Thao, Pham Thanh Cong, J. Phys. Conf. Ser. 200 (2012) 72020.

[6] T. S. Santos, J. S. Moodera, K. V. Raman, E. Negusse, J. Holroyd, J. Dvorak, M. Liberati, Y. U. Idzeda, and E. Arenholz, Phys. Rev. Lett. 101 (2008) 147201.

[7] N. D. Mermin, H. Wagner, Phys. Rev. B 17 (1966) 1133.

[8] Ezana Negusse, J. Dvorak, J. S. Holroyd, M. Liberati, T. S. Santos, J. S. Moodera, E. Arenholz, and Y. U. Idzerda, J. Appl. Phys. 105 (2009) 07C930. 